\title{
A Novel Approach of Impulsive Signal Extraction for Early Fault Detection of Rolling Element Bearing
}

\author{
Hu Aijun, Lin Jianfeng, Sun Shangfei, and Xiang Ling \\ Department of Mechanical Engineering, North China Electric Power University, Baoding, Hebei Province 071003, China \\ Correspondence should be addressed to Hu Aijun; bdlaohu@126.com
}

Received 13 February 2017; Revised 13 July 2017; Accepted 20 July 2017; Published 31 August 2017

Academic Editor: Mariano Artés

Copyright (C) $2017 \mathrm{Hu}$ Aijun et al. This is an open access article distributed under the Creative Commons Attribution License, which permits unrestricted use, distribution, and reproduction in any medium, provided the original work is properly cited.

\begin{abstract}
The fault signals of rolling element bearing are often characterized by the presence of periodic impulses, which are modulated high-frequency harmonic components. The features of early fault in rolling bearing are very weak, which are often masked by background noise. The impulsiveness of the vibration signal has affected the identification of characteristic frequency for the early fault detection of the bearing. In this paper, a novel approach based on morphological operators is presented for impulsive signal extraction of early fault in rolling element bearing. The combination Top-Hat (CTH) is proposed to extract the impulsive signal and enhance the impulsiveness of the bearing fault signal, and the envelope analysis is applied to reveal the fault-related signatures. The impulsive extraction performance of the proposed CTH is compared with that of finite impulse response filter (FIR) by analyzing the simulated bearing fault signals, and the result indicates that the CTH is more effective in extracting impulsive signals. The method is evaluated using real fault signals from defective bearings with early rolling element fault and early fault located on the outer race. The results show that the proposed method is able to enhance the impulsiveness of early bearing fault signals.
\end{abstract}

\section{Introduction}

Rolling element bearings are the most frequently used components in industries; at the same time, bearing faults represent the most frequent cause for failures in rotational machinery. Detecting bearing faults, especially at early stages, is of paramount practical importance.

Vibration analyzing approach is one of the most common methods for bearing fault detection [1] for bearing fault detection. Numerous techniques have been developed in the time domain [2], the frequency domain $[3,4]$, and timefrequency domain $[5,6]$. Artificial intelligent techniques, fuzzy inference [7], neurofuzzy [8], and ART-Kohonen neural network (ART-KNN) [9], for instance, are introduced to enhance fault diagnosis. Jardine et al. [10] reviewed the developments of diagnostics and prognostics in mechanical systems condition-based maintenance (CBM). However, the vibration signal of the defective bearing is complicated. As the rolling elements strike a local fault on the outer or inner race, high-frequency resonance of the whole structure is excited by the shock. The successive impacts produce a series of impulse responses which may be amplitude modulated as a result of the passage of the fault through the load zone or of the varying transmission path between the impact point and the vibration measurement point. In view of this modulation phenomenon, bearing fault signatures are prone to be found at a high-frequency band, which is commonly in a range of $\mathrm{kHz}$. Therefore, the envelope analysis (or resonant demodulation technique) has been early developed to demodulate the signal to obtain a representation of the impulse train. Considering its effectiveness, simplicity in application, and the low computation, envelope analysis has gained a leading role among the techniques applied for rolling element bearing diagnostics in the last years [11]. However, the feature of rolling bearing early fault is very weak and is often buried in the strong background noises. Signal modulation effect and noise are two major barriers in incipient defect detection for bearing fault diagnosis [5]. The success of the envelope analysis technique highly depends on the selection of the center frequency and bandwidth of the band-pass filter used for demodulation [12]. The spectral kurtosis (SK) [13] and fast kurtogram [14] have 
recently attracted strong interest because of its effectiveness in identifying the fault-dominated frequency band containing the fault-induced resonant frequencies. But the performance of the methods needs to be improved when the signal has low signal-to-noise ratio $[1,5]$. Some new filters, such as Kalman filter [12], general particle filter [15], intelligent filter based on artificial neural networks (ANNs) [16], and filter based on time-frequency analysis [17], are introduced for extracting bearing fault features.

Mathematical morphology (MM) $[18,19]$ is a shape based nonlinear signal processing tool. Morphological filter can be employed to decompose a signal into several physical components according to the geometric characteristics of a certain structural element (SE). Recently MM has been introduced to vibration signal analysis [20] and used as noise filter $[21,22]$ or feature extractor $[23,24]$ in bearing fault detection. These studies have demonstrated the effectiveness of MM method in bearing fault detection. But the application of MM in early fault detection of rolling element bearing is hardly reported.

The vibration signal of the defective bearing is a pulse train stemming from the bearing fault, so the best basis of bearing fault detection is obviously the pulse signal itself [1]. It is indicated that the most powerful bearing diagnostic techniques depend on detecting and enhancing the impulsiveness of the signals [25]. Based on our previous study [26] on the working mechanism of MM operators, a new approach of impulsive signal extraction based on $\mathrm{MM}$ is presented for early fault detection of rolling element bearing in the paper. The combination Top-Hat (CTH) is proposed and taken as an extractor to enhance the impulsiveness of the early bearing fault signal; then the envelope analysis is applied to the extracted signal for further demodulation.

The paper is organized as follows. The fundamental theory of MM is described and a new morphological filter is defined in Section 2. The impulsive signal extraction property of the proposed morphological filter is discussed and compared with FIR in Section 3. In Section 4, the proposed approach is verified using the bearing fault signals obtained from two independent experiments. Section 5 presents some final remarks and conclusions.

\section{Morphological Filter}

MM is a theory firstly developed to extract or enhance geometric features from digital images. Its mathematical origins root in set theory, convex analysis, lattice algebra, and integral and stochastic geometry. The basic operators of MM include dilation, erosion, opening, and closing. The definitions of these are presented as follows.

Let $f(n)$ represent a one-dimensional signal and the definition domain is $F=(0,1, \ldots, N-1)$. Let $g(m)$ denote a SE, defined over a domain $G=(0,1, \ldots, M-1)$, and $N \geq M$.

The erosion of $f(n)$ by $g(m)$, denoted by $(f \Theta g)$, is defined as

$$
\begin{aligned}
(f \Theta g)(n)=\min [f(n+m)-g(m)] & \\
& m \in 0,1, \ldots, M-1 .
\end{aligned}
$$

Dilation of $f(n)$ by $g(m)$, denoted by $(f \oplus g)$, is defined as

$$
\begin{aligned}
(f \oplus g)(n)=\max [f(n-m)+g(m)] & \\
& m \in 0,1, \ldots, M-1 .
\end{aligned}
$$

The opening, denoted by $(f \circ g)$, and closing, denoted by $(f \bullet g)$, are defined as

$$
\begin{aligned}
& (f \circ g)(n)=(f \Theta g \oplus g)(n), \\
& (f \circ g)(n)=(f \oplus g \Theta g)(n) .
\end{aligned}
$$

The closing and opening operator can be applied to smooth positive and negative impulses, respectively. In order to restrain bidirectional impulses, opening-closing (OC) and closing-opening (CO) filters are composed, defined as

$$
\begin{aligned}
& \mathrm{OC}(f(n))=(f \circ g \circ g)(n), \\
& \mathrm{CO}(f(n))=(f \circ g \circ g)(n) .
\end{aligned}
$$

$\mathrm{OC}$ and $\mathrm{CO}$ can filter positive and negative impulse noise together, but statistic bias existed. The output magnitude of the OC becomes small and the output magnitude of the $\mathrm{CO}$ is large. So the average value of the two operators, which is named combination morphological filter (CMF), has been proposed. The output signal of the CMF is

$$
\operatorname{CMF}(f(n))=\frac{[\operatorname{CO}(f(n))+\operatorname{OC}(f(n))]}{2} .
$$

The CMF is widely used in signal denoising [27] and it can approach the original signal very well. According to morphological theory, the gauge point of peaks on the original signal can be obtained by subtracting the opening processed signal from the original signal. This process is called white Top-Hat (WTH). Similarly, the gauge point of valleys can be obtained by subtracting the original signal from the closing processed signal, which is called Black Top-Hat (BTH).

$$
\begin{aligned}
\operatorname{WTH}(f(n)) & =f(n)-(f \circ g)(n), \\
\operatorname{BTH}(f(n)) & =(f \circ g)(n)-f(n) .
\end{aligned}
$$

In order to extract bidirectional impulsive signals at the same time, similar to the definition of Top-Hats, the combination Top-Hat (CTH) is proposed and defined as follows:

$$
\mathrm{CTH}(f(n))=f(n)-\operatorname{CMF}(f(n)) .
$$

The CTH has the characteristics of Top-Hat and CMF, and it is used as an impulsive signal extractor. The following case will verify its property.

\section{Impulsive Signal Extraction Property of CTH}

In this section, the bearing fault signal is simulated first. With the simulated signal, the impulsive signal extraction property of the proposed $\mathrm{CTH}$ is proved by comparing with the FIR filter. 


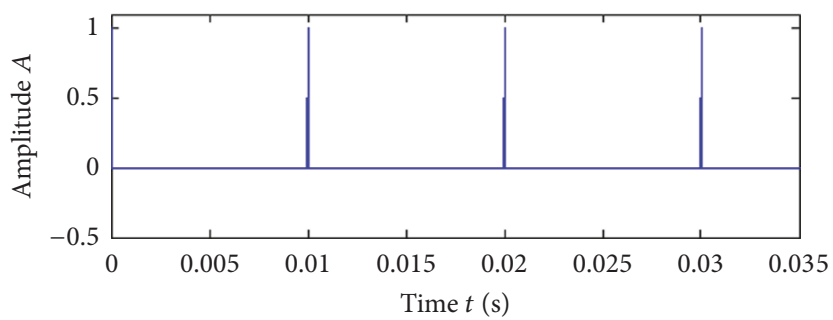

(a)

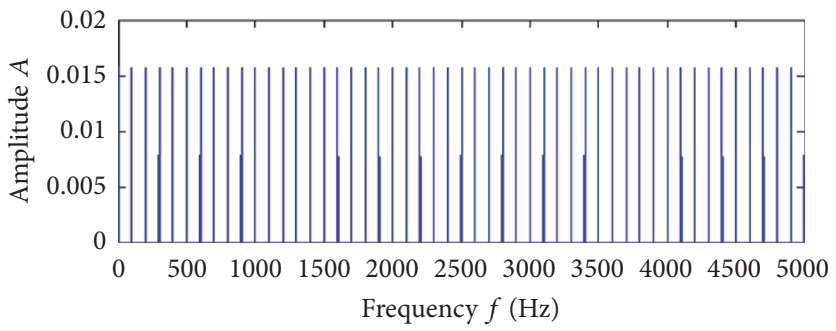

(b)

FIGURE 1: Simulated periodic impulses: (a) time domain waveform and (b) FFT spectrum.

3.1. Simulation of the Bearing Fault Signal. The repetitive impacts produced by a localized bearing defect can be described as a train of Dirac delta functions $\delta(t)$ with the period $T_{d}$, expressed as [28]

$$
x(t)=\sum_{k=0}^{N} A_{k} \delta\left(t-k T_{d}\right),
$$

where $A_{k}$ is the amplitude of the impact. $T_{d}$ is the reciprocal of the characteristic ball pass frequency of a rolling element bearing, depending on the type of the defect. In the simulation, $A_{k}=1, T_{d}$ is set to 0.01 seconds.

When an impulse is applied to the rolling element bearing, the structural resonances will be excited. If only one natural frequency is excited, the impulsive responses can be expressed by the following function:

$$
s(t)=B e^{-\xi 2 \pi f_{0} t} \sin \left(2 \pi f_{0} t\right),
$$

where $B$ is the amplitude of the response, set to $1 ; \xi$ is the attenuation factor, equal to 0.05 , and $f_{0}$ is the natural frequency of the bearing component, set to $2200 \mathrm{~Hz}$.

Referring to (8)-(9), the simulated bearing fault signal $y(t)$ arising from a localized fault can be expressed by

$$
y(t)=\sum_{k=0}^{N}\left[A_{k} \delta\left(t-k T_{d}\right) \otimes s(t)\right],
$$

where the symbol $\otimes$ denotes convolution.

The sampling frequency is $12800 \mathrm{~Hz}$, and a total of 8192 samples are used for FFT analysis. The simulated train of the period impacts and bearing fault signal are shown as Figures 1 and 2 , respectively.

Figure 1(b) shows that the spectrum of the periodic impulses is distributed across the whole frequency range and presented as a series harmonics. The spectrum of the impulsive responses, Figure 2(b), is also expanded in a wide frequency band due to the periodicity of the responses. The results are common in the bearing fault study; however, it emphasizes here that the wide distributed harmonics, including the low frequency components in the spectrum, are produced by the periodicity of the impacts or the impulsive responses. Obviously, the original impacts or the impulsive responses cannot be recovered by any frequency-domain based (low-pass, band-pass, or high-pass) filters, unless the whole frequency band is taken as the filter band.

3.2. Impulsive Signal Extraction. As the rolling elements strike a local fault on the outer or inner race, a series of impulse responses are produced. Therefore, the best basis of bearing fault detection is obviously the pulse signal itself and the most powerful bearing diagnostic techniques depend on detecting and enhancing the impulsiveness of the signals. The impulsive signal extraction property of the CTH is compared with that of finite impulse response filter (FIR) in the section.

The traditional digital filters, infinite impulse response filter (IIR) and finite impulse response filter (FIR), are often used in vibration signal processing. For example, the FIRs are adopted to compose the filter bank in fast kurtogram (FK) algorithm [14] and high-resolution spectral analysis [29], respectively. The FIR filter with a standard Kaiser window [29] and CTH are taken to process the same signal in context for the comparison study.

Different morphological operators have been used in vibration signal processing. It is demonstrated in our previous work that the CTH presents high-pass filter property. And the quantitative relationship between the SE length and cutoff frequency is supplied [26]. Therefore, the SE length can be selected based on the filter property. More detail about the properties of morphological operators, selection principle of morphological filter, and structural element (SE) length can be found in [26]. The CTH is adopted using a flat 


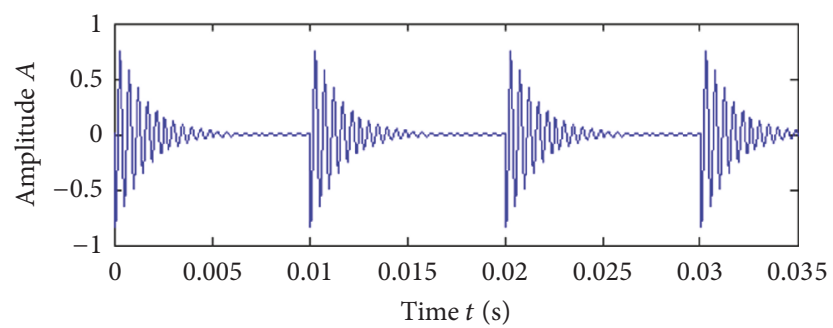

(a)

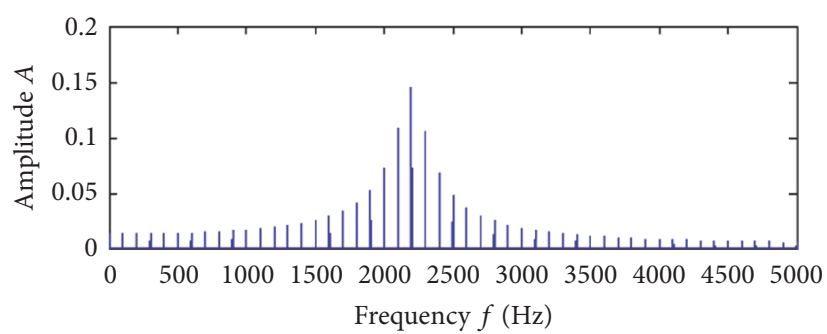

(b)

FIGURE 2: Simulated bearing fault signal: (a) time domain waveform and (b) FFT spectrum.

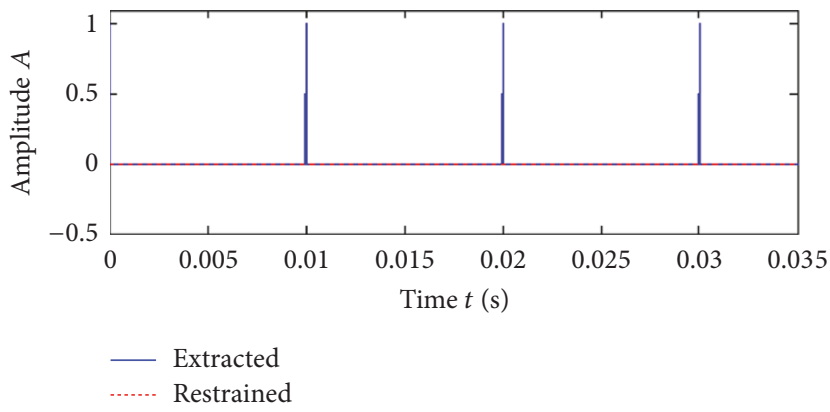

(a)

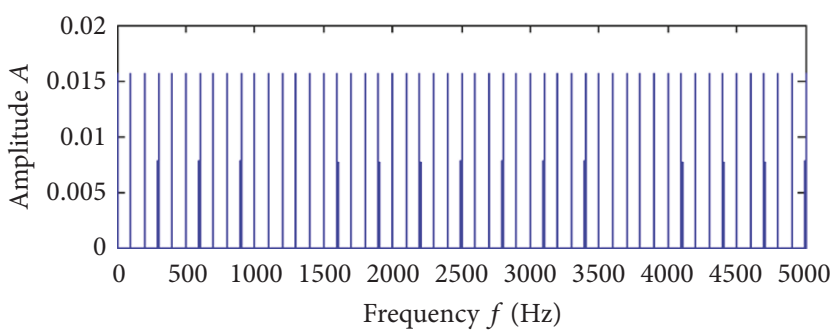

(b)

FIGURE 3: Periodic impacts extraction result of CTH: (a) time domain waveform and (b) FFT spectrum.

SE in the following simulation to evaluate the impulsive signal extraction property of MF. The flat (zero) SEs are selected because they present the simplest SE. The only parameter which must be selected for their application is their corresponding length $L$. After CTH processing, the passed signal is the extraction result and the blocked part is called restrained signal here. The simple signal defined in (8) is analyzed first. The SE length is set to 2; the extraction result of CTH is shown in Figure 3. The restrained signal of the process is shown as red line in Figure 3(a). Referring to Figure 1, the periodic impacts are extracted completely. It means that all the impulses can be extracted or removed from the original signal even with the shortest SE by CTH. This benefited from the geometric based signal process property of MM operator. The components which are shorter than the length of the SE are separated from the original signal.

The filter results of the periodic impacts using a highpass FIR are shown in Figure 4, where the cutoff frequency is set to $2000 \mathrm{~Hz}$. The original signal is also displayed in Figure 4(a) (in red dash line) for comparison. As pointed out in Section 3.1, the periodic impulse cannot be recovered by FIR due to the distributional spectrum. Ignoring the time 


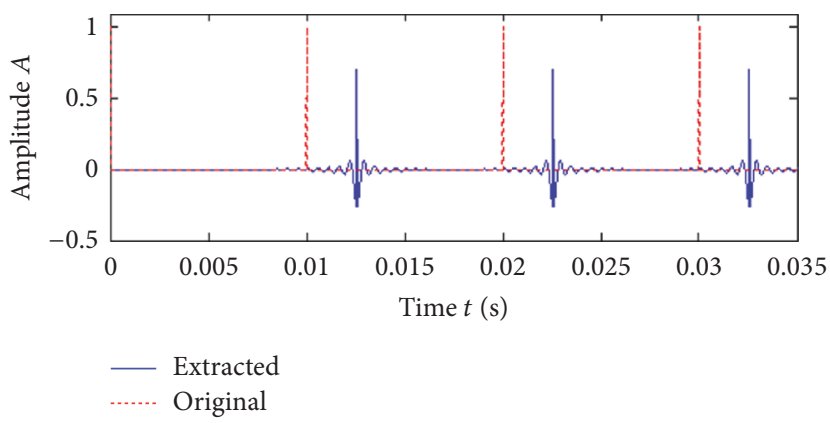

(a)

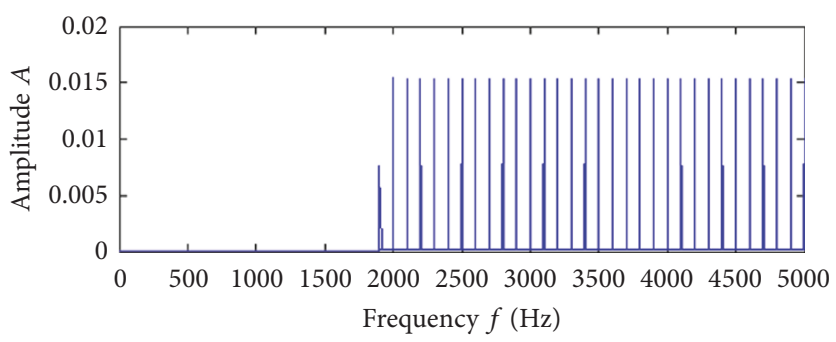

(b)

FIGURE 4: Periodic impacts result of FIR: (a) time domain waveform and (b) FFT spectrum.

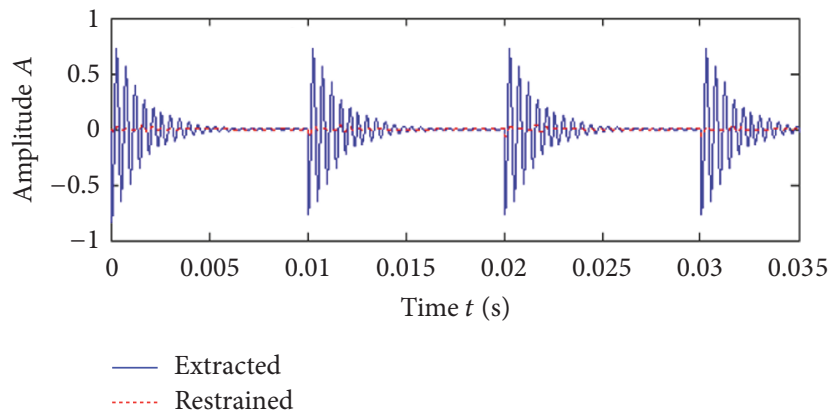

(a)

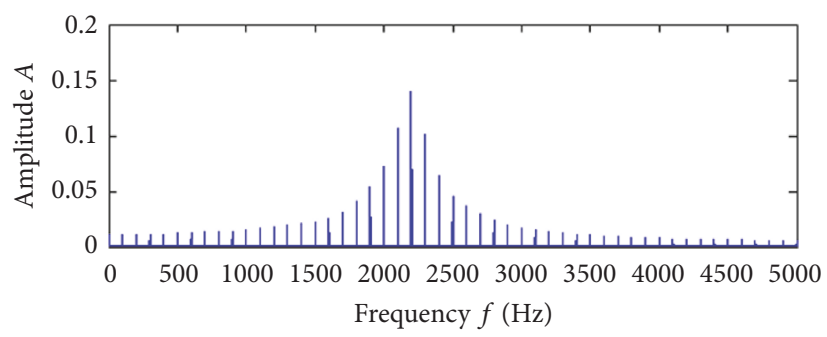

(b)

FIGURE 5: Bearing fault signal extraction result of CTH: (a) time domain waveform and (b) FFT spectrum.

delay, the oscillated components are added to the original impulsive, and the magnitude of the original impulse is reduced. Although the filter results are acceptable in terms of a high-pass filter, for the purpose of impacts extraction, only part of the periodic impulse can be extracted by the FIR.

Then the simulated bearing fault signal shown as Figure 2 is analyzed. The extraction result of CTH is shown in Figure 5, where the SE length is 4 . Figure 5 shows that the time waveform and the spectrum of the extracted signal are almost the same as in Figure 2; thus, the impulsive responses are well extracted. The natural frequency is set to $2200 \mathrm{~Hz}$ in the simulated bearing fault signal, so the band-pass FIR is ranged in $1900 \mathrm{~Hz}-2500 \mathrm{~Hz}$, and the central frequency is set to $2200 \mathrm{~Hz}$. The extraction result of simulated bearing fault signal by the FIR is shown in Figure 6. The start point of the time axis in Figure 6(a) is set to $0.22 \mathrm{~s}$ due to the time delay 


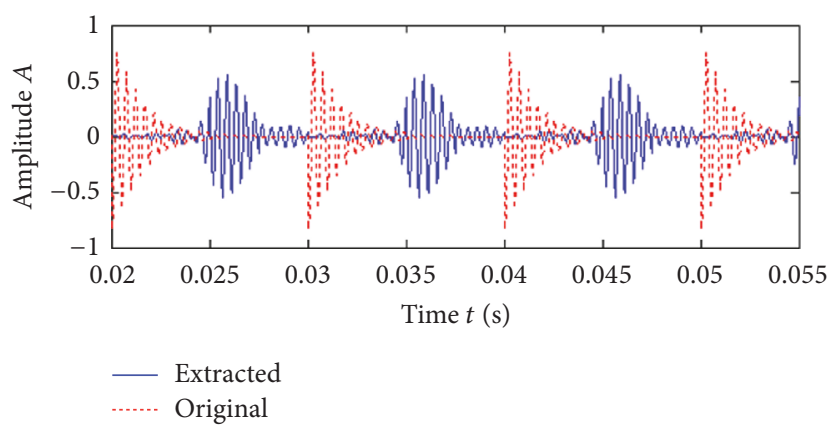

(a)

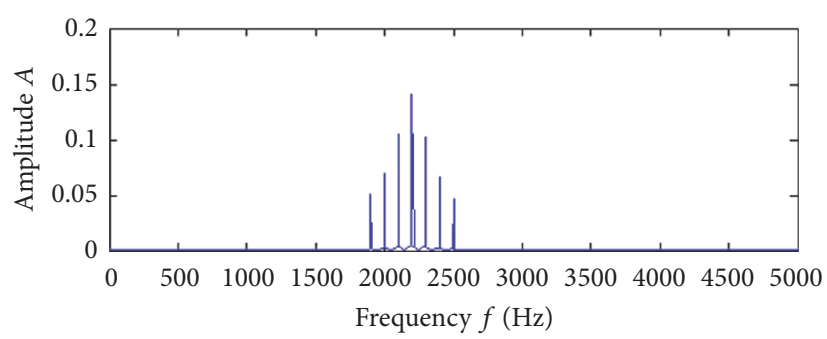

(b)

FIGURE 6: Bearing fault signal extraction result of FIR: (a) time domain waveform and (b) FFT spectrum.

produced by the FIR, and the original signal is also displayed in red dash line for comparison. Figure 6(b) shows that the peaks within the pass-band of the designed FIR are reserved; however, the time waveform in Figure 6(a) is changed when comparing with the original signal. The main reason is that only part of the distributional spectrum can be extract by the FIR.

Kurtosis is one of most used indicators in the condition monitoring of rolling element bearings. A good surface finish bearing has a theoretical kurtosis of 3, and when the surface finish deteriorates, value of kurtosis increases. The kurtosis index is also chosen as a direct measure of the "impulsiveness" of the signal in many signal processing tools, band-stop filtering algorithm [1], band kurtosis, spectral kurtosis, and kurtogram, for instance [30]. Therefore, the kurtosis of the original signal and the extracted signal is calculated to evaluate the impulsiveness of the signal.

The kurtosis results are listed in Table 1. It indicates that the kurtosis of the CTH extracted signal is equal or very close to that of the original signal. That means that the impulsive signals are almost extracted from the original signal. At the same time, the kurtosis of the FIR extracted signal is greatly decreased, which means that only part of impulsive signals are extracted. From the simulation results, it can be seen that the periodic impulsive responses are well extracted and the impulsiveness of the vibration signal is enhanced by the $\mathrm{CTH}$ processing.

\section{Application in Early Bearing Fault}

In this section, we evaluate the effectiveness of the provided method for early bearing fault detection. The vibration signals of two experiments are analyzed. The early bearing fault is
TABLE 1: Kurtosis results.

\begin{tabular}{lccc}
\hline Simulated signal & Original & CTH extracted & FIR extracted \\
\hline Train of Dirac & 126.01 & 126.01 & 67.57 \\
Bearing fault & 10.25 & 9.79 & 6.33 \\
\hline
\end{tabular}

considered as early time of the damage (case 1) or small fault size (case 2). Both the early bearing faults cannot be detected directly using envelope analysis.

4.1. Case 1: Early Fault Signal Analysis of Outer Race. The first data was generated by the NSF I/UCR Center for Intelligent Maintenance Systems, University of Cincinnati. Bearing runto-failure tests are performed under normal load conditions on a specially designed test rig. The test rig is shown in Figure 7. The bearing test rig hosts four test bearings on one shaft. The shaft is driven by an AC motor and coupled by rub belts. The rotation speed was kept constant at $2000 \mathrm{rpm}$. A radial load of $6000 \mathrm{lbs}$. is added to the shaft and bearing by a spring mechanism. All the bearings are force lubricated. A magnetic plug installed in the oil feedback pipe collects the debris from the oil as an evidence of bearing degradation. Four Rexnord ZA-2115 double row bearings were installed on the shaft. The vibration data length is 20480 points with the sampling rate set at $20 \mathrm{kHz}$. More detailed information about this experiment can be found in the literature [31].

The data set was collected from February 12, 2004, 10:32:39, to February 19, 2004, 06:22:39. The experiment lasted a total of 9840 minutes. At the end of the test-to-failure experiment, outer race failure occurred in bearing 1 . The ball pass frequency of the outer race (BPFO) is $236.4 \mathrm{~Hz}$. Figure 8 depicts the kurtosis of bearing 1 for the entire life cycle. 

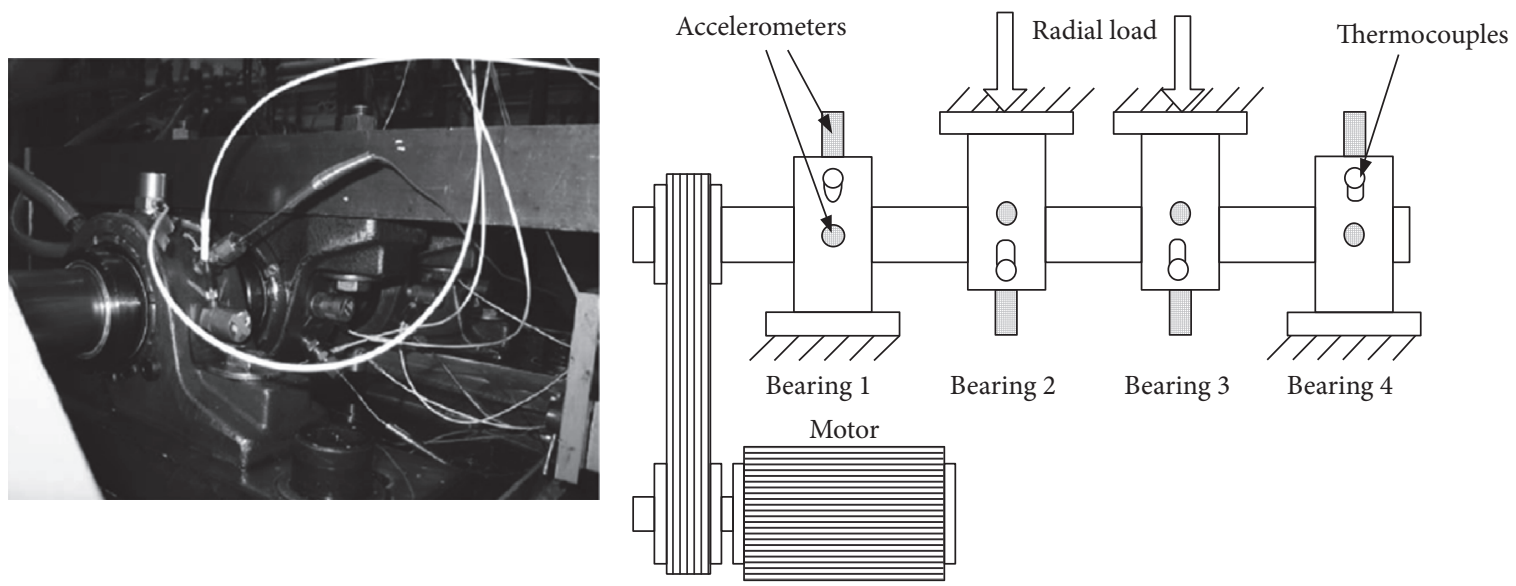

FIGURE 7: Bearing test rig and sensor placement illustration.

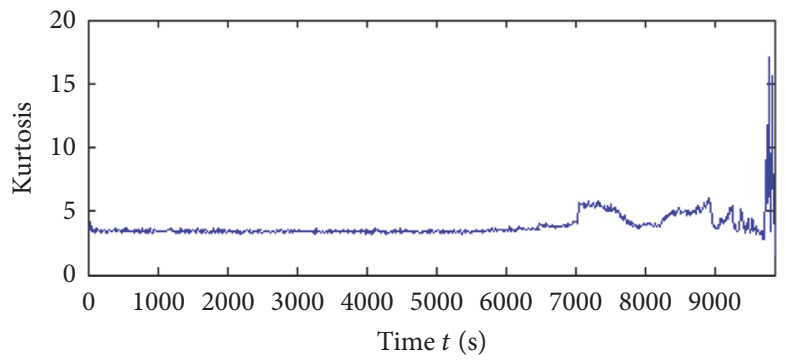

FIgURE 8: The kurtosis of bearing 1 for the whole life cycle.

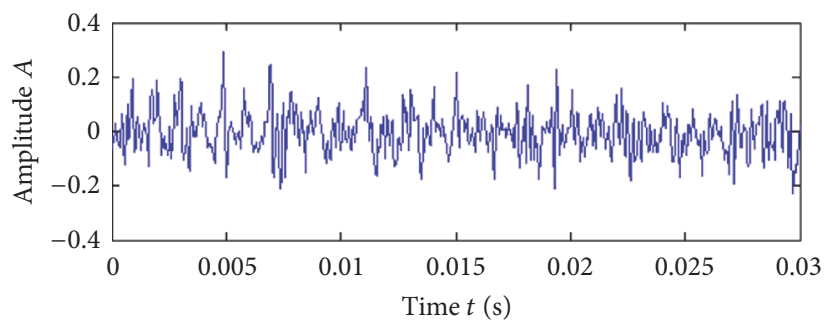

(a)

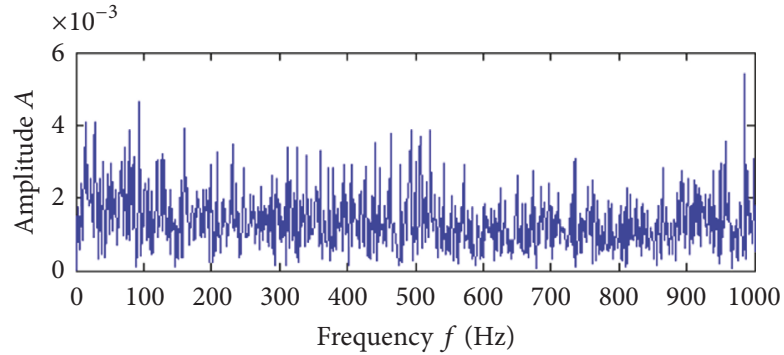

(b)

FIGURE 9: Vibration signal of early outer race fault: (a) time waveform and (b) envelope spectrum.

Figure 8 indicates that the period from 0 to 6900 minutes is the early stage of the damage propagating process in the entire life cycle.

It is reported that the early bearing outer race faults occurring at file number $533(2004.02 .16 .03 .12 .39)$ is detected
[32]. Here, the data collected one day earlier, whose file number is 527 (2004.02.16.02.12.39), is adopted for further analysis. Figure 9 presents the vibration signal and its envelope spectrum. The kurtosis of the original signal is calculated to 3.39 , and there is no clear sign of periodic impulse in the 


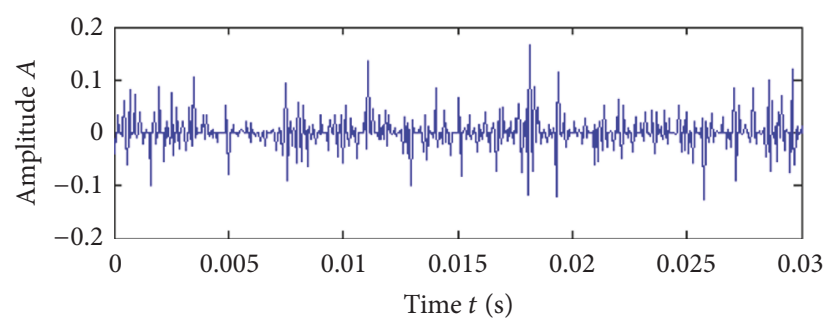

(a)

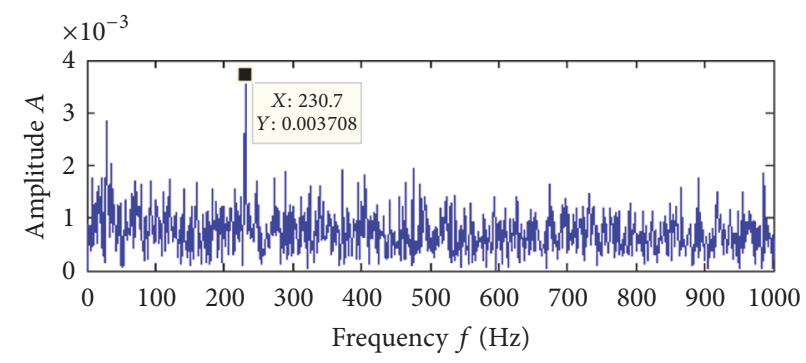

(b)

FIGURE 10: Extraction result of early outer race fault: (a) time waveform and (b) envelope spectrum.

time waveform. The outer race fault characteristic frequency cannot be found either in the FFT spectrum (not shown) or in the envelope spectrum (Figure 9(b)).

The proposed method is adopted to extract the impulsive feature from the early fault signal shown above. The CTH operator is applied to the bearing fault signal, and then the envelope analysis is conducted. The analysis results are shown in Figure 10. It shows that the impulsiveness is enhanced and the kurtosis of the extracted signal is increased to 9.69. The $230.7 \mathrm{~Hz}$ component, which is the actual outer race fault frequency [26], is prominent in the envelop spectrum. The early outer race fault is detected.

\subsection{Case 2: Slight Fault Signal Analysis of Rolling Element.} The second experimental data of rolling bearing are collected from the Case Western Reserve University (CWRU) Bearing Data Centre Website [33]. The test rig is shown in Figure 11. The bearings used at the fan end are the deep groove ball bearing 6203-2RS JEM SKF. Single point faults were introduced to the test bearings using electrodischarge machining with fault diameters of $0.1778 \mathrm{~mm}$ ( 7 mils), $0.3556 \mathrm{~mm}$ (14 mils),

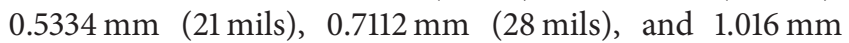
(40 mils). The signal of the slight rolling element fault bearing with $0.1778 \mathrm{~mm}$ fault size is chosen for the following analysis.

The original vibration signal and its spectrum of the defective bearing with slight rolling element fault are shown in Figure 12. The measurement is performed with sampling frequency of $12 \mathrm{kHz}$ at the rotating speed of $1777 \mathrm{rpm}$ and $1 \mathrm{HP}$ load. The calculated rolling element fault characteristic frequency is $118.09 \mathrm{~Hz}$. Figure $12(\mathrm{a})$, the time waveform, shows that the impulsive feature of the slight rolling element defect is not significant. The calculated kurtosis of the signal is 3.71. Figure 13(a), the local FFT spectrum, shows that the fault characteristic frequency cannot be found in the spectrum.

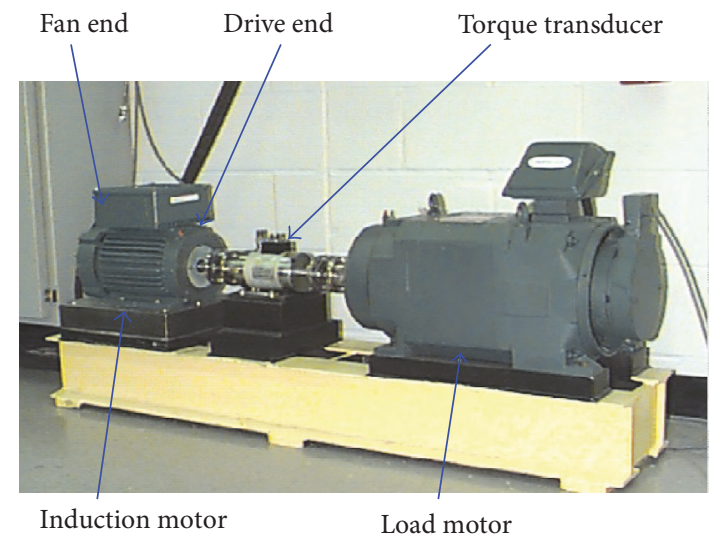

FIGURE 11: The bearing test rig of CWRU.

The envelop spectrum of the vibration signal is shown as Figure 13(b). Although the frequency $117.9 \mathrm{~Hz}$ is presented by the envelop analysis, the fault characteristic frequency has very low amplitude and is hidden in other harmonics. Therefore, this slight rolling element fault can hardly be detected through conventional frequency-domain methods.

The proposed method is employed to extract the impulsive feature from the weak rolling element fault signal. The $\mathrm{CTH}$ operator is applied to the bearing signal with rolling element fault. The extracted results are shown in Figure 14(a). It shows that the transient impulse is enhanced and the kurtosis of the extracted signal is increased to 10.57. The fault characteristic frequency, $117.9 \mathrm{~Hz}$ is clearly visible in the envelop spectrum, as shown in Figure 14(b). The weak impulsive feature is effectively enhanced by the proposed method and the slight rolling element fault is detected. 


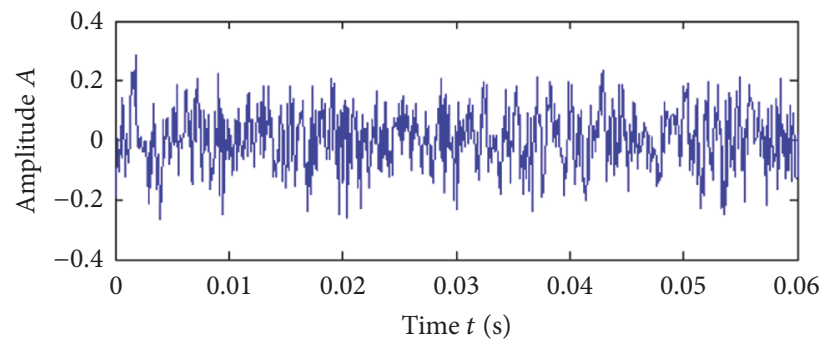

(a)

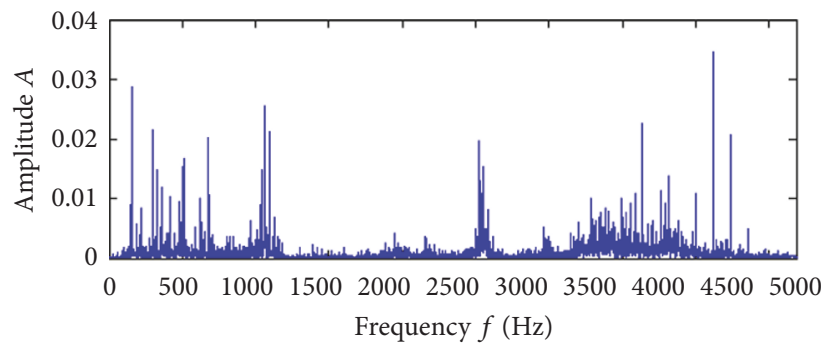

(b)

FIGURE 12: Vibration signal of slight rolling element fault: (a) time waveform and (b) the FFT spectrum.

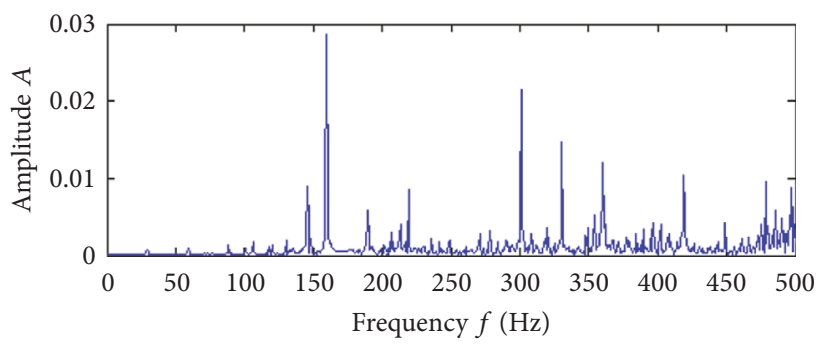

(a)

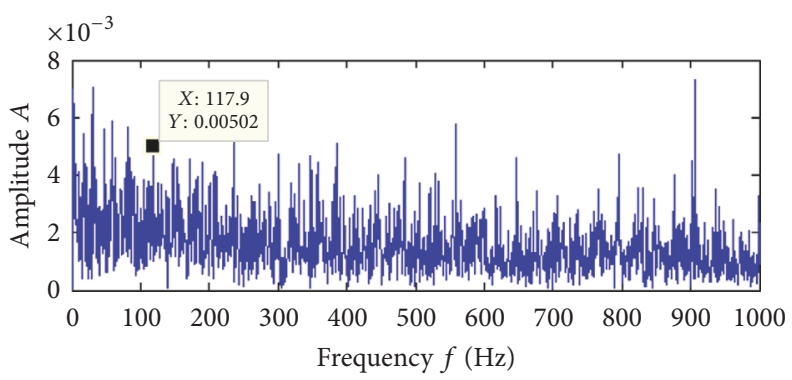

(b)

FIGURE 13: Spectra of slight rolling element fault: (a) local spectrum and (b) envelope spectrum.

\section{Conclusion}

The features of early fault in rolling bearing are very weak and usually have low energy level. Aiming to the impulsiveness enhancement of the early bearing fault signal, the mathematical morphological operator, combination Top-Hat $(\mathrm{CTH})$ is proposed to extract the impulsive signal. Then the envelope analysis is applied to reveal the fault-related signatures. Comparing with the FIR, the advantage of CTH in impulsive feature extraction is discussed. It shows that the periodic impulsive signal can be well extracted by the CTH processing and the impulsiveness of bearing fault signal is enhanced. The proposed method is verified by using real fault signals from defective bearings with early rolling element fault and early fault located on the outer race. The early bearing faults are effectively detected. 


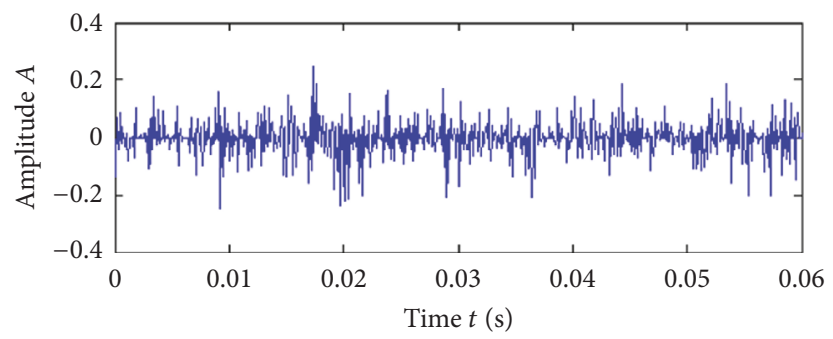

(a)

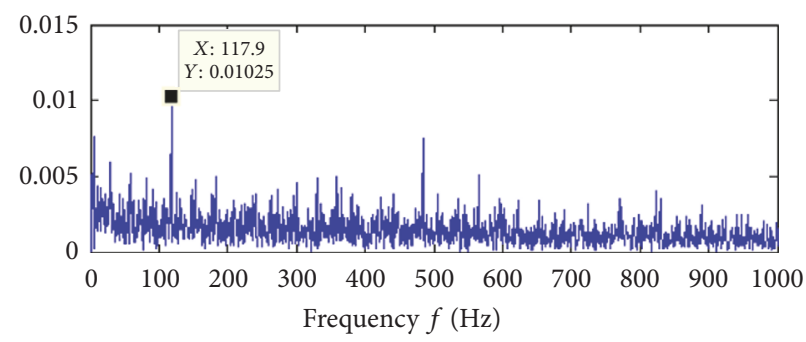

(b)

FIGURE 14: Extraction result of slight rolling element fault: (a) time waveform and (b) envelope spectrum.

\section{Conflicts of Interest}

The authors declare that they have no conflicts of interest.

\section{Acknowledgments}

This work was supported by the National Natural Science Foundation of China (Grant nos. 51475164 and 51675178).

\section{References}

[1] Y. Zhang, M. Liang, C. Li, and S. Hou, "A joint kurtosisbased adaptive bandstop filtering and iterative autocorrelation approach to bearing fault detection," Journal of Vibration and Acoustics, Transactions of the ASME, vol. 135, no. 5, Article ID 051026, 2013.

[2] J. Yu, "Local and nonlocal preserving projection for bearing defect classification and performance assessment," IEEE Transactions on Industrial Electronics, vol. 59, no. 5, pp. 2363-2376, 2012.

[3] W. Sui, S. Osman, and W. Wang, "An adaptive envelope spectrum technique for bearing fault detection," Measurement Science and Technology, vol. 25, no. 9, p. 095004, 2014.

[4] I. Antoniadis and G. Glossiotis, "Cyclostationary analysis of rolling-element bearing vibration signals," Journal of Sound and Vibration, vol. 248, no. 5, pp. 829-845, 2001.

[5] Y. Wang, G. Xu, L. Liang, and K. Jiang, "Detection of weak transient signals based on wavelet packet transform and manifold learning for rolling element bearing fault diagnosis," Mechanical Systems and Signal Processing, vol. 54-55, pp. 259-276, 2015.

[6] F. Jiang, Z. Zhu, W. Li, G. Chen, and G. Zhou, "Robust condition monitoring and fault diagnosis of rolling element bearings using improved EEMD and statistical features," Measurement Science and Technology, vol. 25, no. 2, Article ID 025003, 2014.

[7] X. S. Lou and K. A. Loparo, "Bearing fault diagnosis based on wavelet transform and fuzzy inference," Mechanical Systems and Signal Processing, vol. 18, no. 5, pp. 1077-1095, 2004.
[8] G. N. Marichal, M. Artés, J. C. García Prada, and O. Casanova, "Extraction of rules for faulty bearing classification by a NeuroFuzzy approach," Mechanical Systems and Signal Processing, vol. 25, no. 6, pp. 2073-2082, 2011.

[9] B. S. Yang, T. Han, and Y. Kim, "Integration of ART-Kohonen neural network and case-based reasoning for intelligent fault diagnosis," Expert Systems with Applications, vol. 26, no. 3, pp. 387-395, 2004.

[10] A. K. S. Jardine, D. Lin, and D. Banjevic, "A review on machinery diagnostics and prognostics implementing condition-based maintenance," Mechanical Systems and Signal Processing, vol. 20, no. 7, pp. 1483-1510, 2006.

[11] P. Borghesani, R. Ricci, S. Chatterton, and P. Pennacchi, "A new procedure for using envelope analysis for rolling element bearing diagnostics in variable operating conditions," Mechanical Systems and Signal Processing, vol. 38, no. 1, pp. 23-35, 2013.

[12] S. Khanam, J. K. Dutt, and N. Tandon, "Extracting rolling element bearing faults from noisy vibration signal using kalman filter," Journal of Vibration and Acoustics, Transactions of the ASME, vol. 136, no. 3, Article ID 031008, 2014.

[13] Y. Wang and M. Liang, "An adaptive SK technique and its application for fault detection of rolling element bearings," Mechanical Systems and Signal Processing, vol. 25, no. 5, pp. 1750-1764, 2011.

[14] J. Antoni, "Fast computation of the kurtogram for the detection of transient faults," Mechanical Systems and Signal Processing, vol. 21, no. 1, pp. 108-124, 2007.

[15] D. Wang and Q. Miao, "Some improvements on a general particle filter based bayesian approach for extracting bearing fault features," Journal of Vibration and Acoustics, Transactions of the ASME, vol. 137, no. 4, Article ID 041016, 2015.

[16] J. Zarei, M. A. Tajeddini, and H. R. Karimi, "Vibration analysis for bearing fault detection and classification using an intelligent filter," Mechatronics, vol. 24, no. 2, pp. 151-157, 2014.

[17] B. Q. Chen, Z. S. Zhang, Y. Y. Zi, Z. J. He, and C. Sun, "Detecting of transient vibration signatures using an improved fast spatialspectral ensemble kurtosis kurtogram and its applications to 
mechanical signature analysis of short duration data from rotating machinery," Mechanical Systems and Signal Processing, vol. 40, no. 1, pp. 1-37, 2013.

[18] P. Maragos and R. W. Schafer, "Morphological filters. I. Their set-theoretic analysis and relations to linear shift-invariant filters," Institute of Electrical and Electronics Engineers. Transactions on Acoustics, Speech, and Signal Processing, vol. 35, no. 8, pp. 1153-1169, 1987.

[19] P. Maragos and R. W. Schafer, "Morphological filters. II. Their relations to median, order-statistic, and stack filters," Institute of Electrical and Electronics Engineers. Transactions on Acoustics, Speech, and Signal Processing, vol. 35, no. 8, pp. 1170-1184, 1987.

[20] N. G. Nikolaou and I. A. Antoniadis, "Application of morphological operators as envelope extractors for impulsive-type periodic signals," Mechanical Systems and Signal Processing, vol. 17, no. 6, pp. 1147-1162, 2003.

[21] L. J. Meng, J. W. Xiang, Y. X. Wang, Y. Y. Jiang, and H. F. Gao, "A hybrid fault diagnosis method using morphological filter-translation invariant wavelet and improved ensemble empirical mode decomposition," Mechanical Systems and Signal Processing, vol. 50-51, pp. 101-115, 2015.

[22] A. S. Raj and N. Murali, "Early classification of bearing faults using morphological operators and fuzzy inference," IEEE Transactions on Industrial Electronics, vol. 60, no. 2, pp. 567-574, 2013.

[23] J. Wang, G. Xu, Q. Zhang, and L. Liang, "Application of improved morphological filter to the extraction of impulsive attenuation signals," Mechanical Systems and Signal Processing, vol. 23, no. 1, pp. 236-245, 2009.

[24] Z. W. Chen, N. Gao, W. Sun et al., "A signal based triangular structuring element for mathematical morphological analysis and its application in rolling element bearing fault diagnosis," Shock and Vibration, vol. 2014, Article ID 590875, 16 pages, 2014.

[25] R. B. Randall and J. Antoni, "Rolling element bearing diagnostics-a tutorial," Mechanical Systems and Signal Processing, vol. 25, no. 2, pp. 485-520, 2011.

[26] A. Hu and L. Xiang, "Selection principle of mathematical morphological operators in vibration signal processing," Journal of Vibration and Control, vol. 22, no. 14, pp. 3157-3168, 2016.

[27] F. F. Costa, A. J. Sguarezi Filho, C. E. Capovilla, and I. R. S. Casella, "Morphological filter applied in a wireless deadbeat control scheme within the context of smart grids," Electric Power Systems Research, vol. 107, pp. 175-182, 2014.

[28] T. I. Patargias, C. T. Yiakopoulos, and I. A. Antoniadis, "Performance assessment of a morphological index in fault prediction and trending of defective rolling element bearings," Nondestructive Testing and Evaluation, vol. 21, no. 1, pp. 39-60, 2006.

[29] A. Garcia-Perez, R. D. J. Romero-Troncoso, E. Cabal-Yepez, and R. A. Osornio-Rios, "The application of high-resolution spectral analysis for identifying multiple combined faults in induction motors," IEEE Transactions on Industrial Electronics, vol. 58, no. 5, pp. 2002-2010, 2011.

[30] P. Borghesani, P. Pennacchi, and S. Chatterton, "The relationship between kurtosis- and envelope-based indexes for the diagnostic of rolling element bearings," Mechanical Systems and Signal Processing, vol. 43, no. 1-2, pp. 25-43, 2014.

[31] H. Qiu, J. Lee, J. Lin, and G. Yu, "Wavelet filter-based weak signature detection method and its application on rolling element bearing prognostics," Journal of Sound and Vibration, vol. 289, no. 4-5, pp. 1066-1090, 2006.
[32] D. Wang and C. Shen, "An equivalent cyclic energy indicator for bearing performance degradation assessment," Journal of Vibration and Control, 2014.

[33] Bearing Data Center - Case Western Reserve University, Cleveland, Ohio, USA, http://www.eecs.case.edu/laboratory/bearing. 


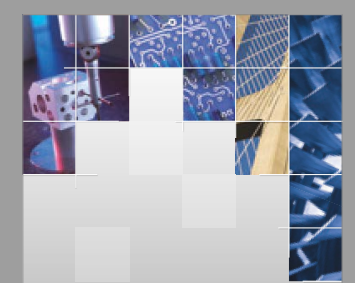

\section{Enfincering}
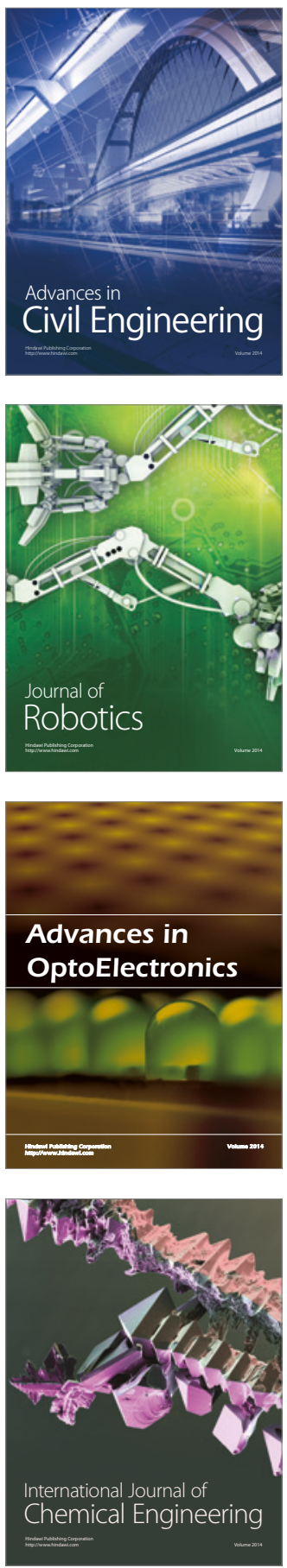

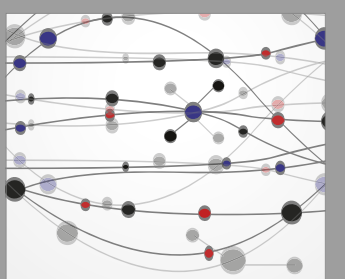

The Scientific World Journal

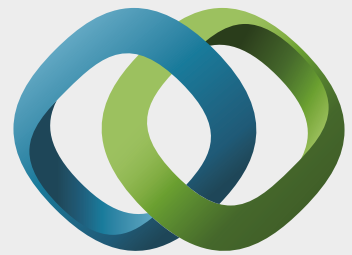

\section{Hindawi}

Submit your manuscripts at

https://www.hindawi.com
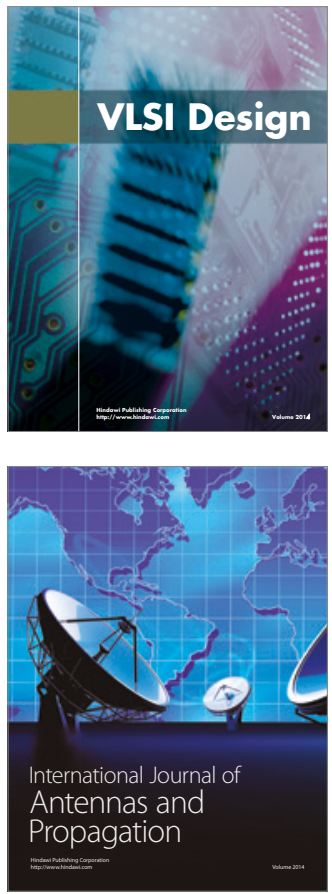

\section{Rotating}

Machinery
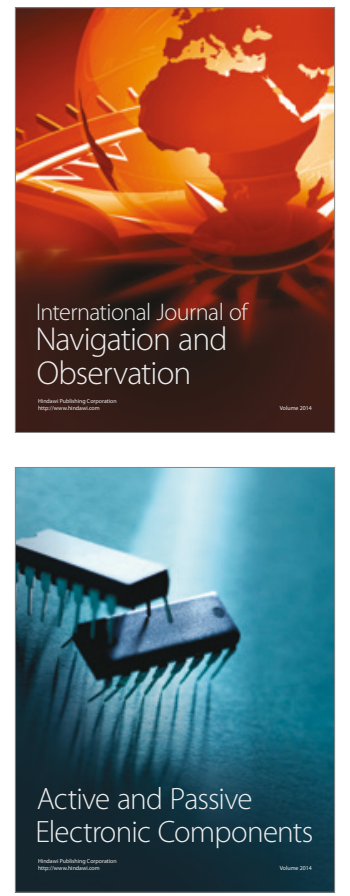
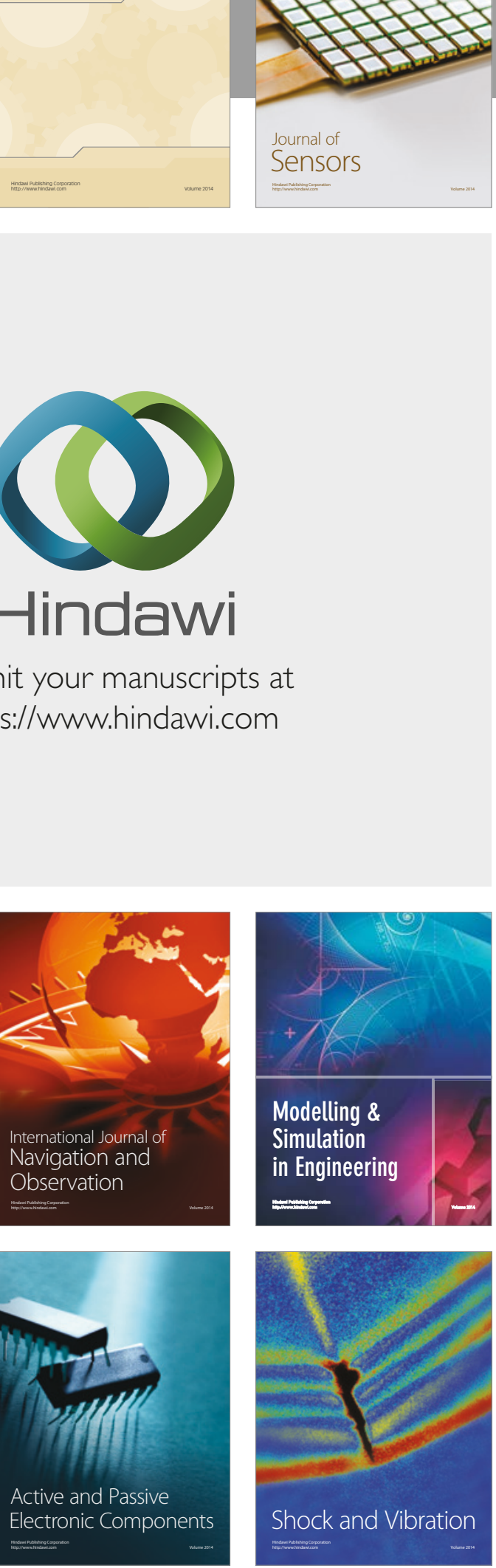
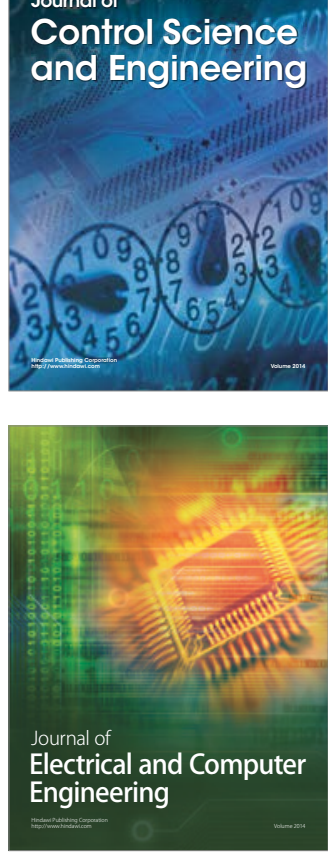

Distributed

Journal of

Control Science

and Engineering
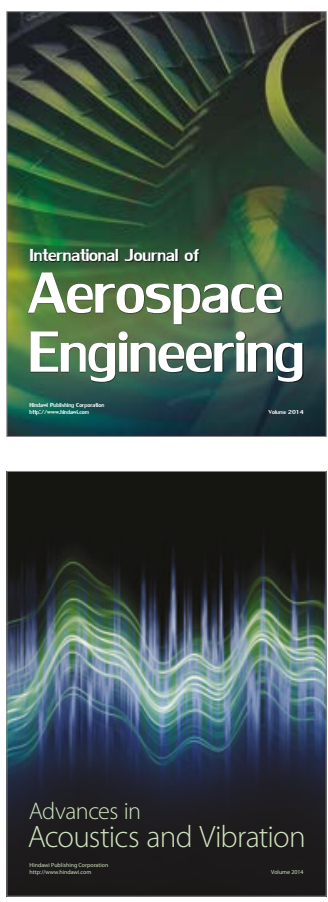

Sensor Networks 